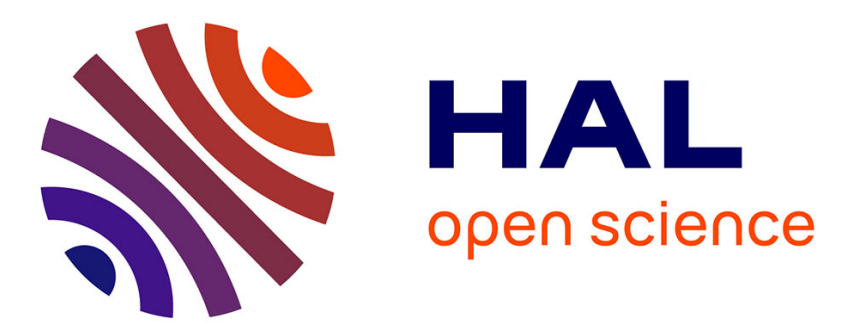

\title{
Les multiples facettes de l'entrepreneuriat scientifique de James Lovelock dans les années 1960-70: développement d'instruments, consultance sur les pollutions et hypothèse Gaïa
}

Régis Briday, Sébastien Dutreuil

\section{To cite this version:}

Régis Briday, Sébastien Dutreuil. Les multiples facettes de l'entrepreneuriat scientifique de James Lovelock dans les années 1960-70: développement d'instruments, consultance sur les pollutions et hypothèse Gaïa. Marché et Organisations, 2019, L'entrepreneuriat scientifique: institutions et innovation, 34, pp.33-60. 10.3917/maorg.034.0033 . hal-02305427

\section{HAL Id: hal-02305427 \\ https://hal.science/hal-02305427}

Submitted on 4 Oct 2019

HAL is a multi-disciplinary open access archive for the deposit and dissemination of scientific research documents, whether they are published or not. The documents may come from teaching and research institutions in France or abroad, or from public or private research centers.
L'archive ouverte pluridisciplinaire HAL, est destinée au dépôt et à la diffusion de documents scientifiques de niveau recherche, publiés ou non, émanant des établissements d'enseignement et de recherche français ou étrangers, des laboratoires publics ou privés. 
LES MULTIPLES FACETTES DE L'ENTREPRENEURIAT SCIENTIFIQUE DE JAMES LOVELOCK DANS LES ANNEES 1960-70 : DÉVELOPPEMENT D'INSTRUMENTS, CONSULTANCE SUR LES POLLUTIONS ET HYPOTHÈSE GÄ̈A

\author{
Régis BRIDAY \\ Laboratoire Techniques, Territoires et Sociétés, Champs-sur-Marne, France \\ regisbriday@yahoo.fr
}

Sébastien DUTREUIL

Aix Marseille Univ, CNRS, Centre Gilles Gaston Granger, Aix-en-Provence, France

seb.dutreuil@gmail.com

\title{
Résumé
}

Chimiste célèbre pour avoir élaboré l'hypothèse Gaïa, James Lovelock (1919-...) se présente comme un «scientifique indépendant» installé dans son laboratoire-maison, retiré dans la campagne anglaise. À rebours de ce récit, les auteurs décrivent son parcours dans les années 1960-70 comme celui d'un «scientifique entrepreneur». Après vingt ans comme ingénieurfonctionnaire dans le domaine biomédical, Lovelock délaisse cette position pour s'établir à son compte. Son expertise dans le domaine de la chromatographie et l'invention d'instruments font de lui un consultant courtisé, aussi bien par de grandes entreprises chimiques et pétrolières que par de prestigieuses institutions scientifiques publiques. De grandes synergies, mais aussi des tensions, s'opèrent entre ses activités d'expert sur des questions environnementales, de consultant pour des entreprises et de théoricien élaborant une nouvelle conception de la Terre et de la vie. Lovelock ne doit plus uniquement être regardé comme l'un des pères des sciences du système Terre et un acteur important des mutations culturelles autour de l'environnement depuis les années 1960, mais également comme une incarnation précoce de scientifique entrepreneur avec un pied dans la recherche publique sur l'environnement et l'autre dans la recherche industrielle.

\section{Mots-clés}

Lovelock ; scientifique entrepreneur ; consultance ; expertise environnementale ; Gaïa

\section{Summary}

James Lovelock (1919-...), a chemist famous for having elaborated the Gaia hypothesis, portrays himself as an "independent scientist" settled in a remote lab-house in the English countryside. Against this narrative, the authors describe Lovelock's life in the 1960s and 1970s as that of a "scientific entrepreneur". After twenty years as an engineer in a civil biomedical laboratory, Lovelock resigned to set up on his own. His skills as an expert in chromatography and as an engineer gifted for inventing chemistry instruments were so renowned that he rapidly became a highly demanded consultant, working both for major chemical and petroleum industries and for prestigious public scientific institutions. Obvious synergies, but also tensions, appear between his activities as an expert on environmental issues, his consulting work for industries, and his theoretical work giving birth to a new conception of Earth and life. Lovelock must not only be eventually regarded as one of the fathers of Earth system science and as an important contributor to cultural mutations linked to the environment from the 1960s onwards, but also as an early embodiment of a scientific entrepreneur with a foot in the world of public environmental research and the other in the world of industry.

\section{Keywords}

Lovelock; scientific entrepreneur; consulting; environmental expertise; Gaia 
Les historiens des sciences ont popularisé les figures de l'inventeur-entrepreneur, de l'ingénieur-entrepreneur et de l'entrepreneur scientifique. Ils ont montré que, depuis le XVIIème siècle, les savants prennent place dans des réseaux qui s'étirent hors les murs du laboratoire et de l'arène scientifique : ils adoptent des stratégies de courtisan, sortent leurs instruments et expériences des laboratoires pour intégrer les milieux fortunés et les quartiers d'affaires, assoient leur prospérité sur le dépôt de brevets, la création et l'entretien de réseaux capitalistiques et marchands, ou encore créent et/ou dirigent des manufactures... tout en étant parfois des experts sur les risques que génèrent leurs activités (Biagioli, 1993 ; Stewart, 1997 ; Galvez-Behar, 2016 ; Massard-Guilbaud, 2003).

Les reconfigurations des politiques industrielles et scientifiques des années 1970 ont fait l'objet d'une attention particulière. Steven Shapin a montré qu'aux États-Unis, « les routines et motifs institutionnels» se transforment pour faire une place à «l'entrepreneuriat scientifique » dans l'agenda de l'universitaire public, notamment en lui permettant de commercialiser ses idées scientifiques en créant une entreprise et/ou de les valoriser par des activités de consultance en gardant un pied à l'université. Si «le rôle du scientifique entrepreneur n'est certes pas complètement nouveau, $\{\ldots\}$ ce n'est qu'à partir des années 1970 environ que l'activité entrepreneuriale commence substantiellement à façonner les appréciations des Américains au sujet du genre de personne que le scientifique est, et au sujet de ce à quoi la vie scientifique peut mener » (Shapin, 2008, pp. 250-252 \& 209-210). Plusieurs auteurs ont en outre montré le rôle spécifique de l'État dans la fabrique d'un entrepreneuriat académique (e.g. Boutillier, 2003).

Dans cet article, nous nous intéressons au cas du chimiste britannique James Lovelock (1919...), célèbre pour avoir élaboré l'hypothèse Gaïa suivant laquelle l'environnement global serait régulé par l'activité des vivants - avec le succès que l'on connait : les transformations que Gaïa a engendrées sur la manière dont nous nous représentons la Terre, la nature et les vivants sont d'une importance telle, que Bruno Latour les a comparées à celles opérées par Galilée (Latour, 2015). Lovelock s'est mis en scène comme "scientifique indépendant » (première section); "scientifique entrepreneur" lui correspondrait davantage. Nous montrons en effet que sa trajectoire biographique dans les milieux scientifiques universitaires et industriels du Royaume-Uni et des États-Unis des années 1960-70 obéit par certains aspects à la logique des scientifiques entrepreneurs que décrit Shapin, qui ont « un pied dans la production de connaissances et l'autre dans la fabrication d'instruments, de services et, finalement d'argent », incarnant en cela - plus ou moins consciemment - « des conduites et des activités qui, au cours du XXème siècle, ont largement été considérées comme en tension, et parfois en opposition » (Shapin, 2008, p. 210). L'entrepreneuriat de Lovelock doit également être appréhendé par-delà ses liens directs à des activités commerciales : son insertion dans la recherche académique comme l'industrie est indissociable de l'élaboration d'un programme scientifique «personnel », l'hypothèse Gaïa, et de sa participation aux débats relatifs aux pollutions environnementales. Nous décrivons d'abord les démarches qui permettent à Lovelock de s'établir comme entrepreneur dans les années 1960 en valorisant ses talents d'inventeur d'instruments (deuxième section). Puis, nous passons en revue les activités concrètes qui occupent le quotidien de Lovelock (troisième section). Enfin, nous détaillons les nombreuses synergies institutionnelles et thématiques entre les activités de Lovelock dans le public et le privé, et proposons quelques perspectives sur les éventuels conflits d'intérêt entre ces activités (quatrième section).

Liste des principales abréviations :

CFC Chlorofluorocarbures

CMA Chemical Manufacturers Association 
DMS Sulfure de diméthyle

ECD Electron Capture Detector

GIEC Groupe d'Experts Intergouvernemental sur l'Évolution du Climat

HP Hewlett-Packard

ICI Imperial Chemical Industries

IGBP International Geosphere-Biosphere Programme

JPL Jet Propulsion Laboratory

MRC Medical Research Council

NCAR National Center for Atmospheric Research

NIMR National Institute for Medical Research

NOAA National Oceanic and Atmospheric Administration

\section{LE RECIT AUTOBIOGRAPHIQUE LOVELOCKIEN EN QUESTION}

À presque cent ans, Lovelock est célébré par la communauté internationale de la chimie et des géosciences, les Britanniques suivent ses positions sur le changement climatique dans leur presse généraliste, les passionnés de science lisent ses essais consacrés à Gaïa.

\section{Les instruments de chromatographie : le nœud de la trajectoire biographique de Lovelock}

Diplômé en chimie à l'Université de Manchester, Lovelock intègre en 1941 le National Institute for Medical Research (NIMR), un institut rattaché au Medical Research Council (MRC). Lovelock y travaille comme ingénieur-chimiste et chercheur dans le domaine biomédical. Il publie des articles sur l'effet de la chaleur ou de la congélation sur les cellules, les tissus et la coagulation sanguine, sur la transmission d'infections, et divers travaux de biochimie. Au NIMR, il côtoie Archer Martin, co-lauréat du Prix nobel de chimie en 1952 pour l'invention de la chromatographie de partage, et figure précoce de la chromatographie en phase gazeuse. Son ancrage au sein de la communauté du NIMR est déterminante. Le talent de Lovelock à inventer des instruments de détection qui, à la sortie de chromatographes, permettent de mesurer des concentrations infimes de composés chimiques - en particulier, le détecteur à ionisation d'argon en 1956 et le détecteur à capture d'électrons (ECD) en 1957 (l'instrument qui jouera le rôle le plus important dans sa carrière scientifique) -, va en effet constituer le nœud central de la trajectoire biographique de Lovelock. Un nœud qui relie la trajectoire de l'ingénieur-développeur d'instruments et celle du scientifique contribuant à des champs aussi divers que la cryobiologie, la biochimie des lipides, la chimie de l'atmosphère, l'océanographie ou la géochimie.

Ainsi, c'est pour ses talents d'ingénieur que Lovelock est contacté en 1961 par le Jet Propulsion Laboratory (JPL) de la NASA afin de travailler comme consultant au développement de chromatographes équipant les robots de la mission Surveyor devant explorer la Lune. Après vingt années passées au NIMR, ce contrat va lui servir de tremplin pour s'établir comme scientifique entrepreneur. Il obtient en sus un poste à l'Université de Houston au Lipid research center (1961-1964). En 1964, il retourne définitivement en Angleterre, à Bowerchalke, un village isolé du sud-ouest. Il devient consultant pour de nombreuses entreprises et institutions et commence à élaborer l'hypothèse Gaïa à partir de la fin des années $1960 .{ }^{1}$

\footnotetext{
${ }^{1}$ Pour une histoire détaillée de l'élaboration et la réception scientifique de l'hypothèse Gaïa, voir (Dutreuil, 2016).
} 


\section{Lovelock se met en scène comme « un scientifique indépendant »}

Conscient de sa place dans l'histoire des sciences et l'histoire environnementale, Lovelock a assidûment délivré un récit de sa propre vie. Il y met en scène la figure du «scientifique indépendant ", comme dans son autobiographie Homage to Gaia : the life of an independent scientist, où il déclare : "La science était et est ma passion et je voulais être libre de la pratiquer sans l'entrave de la direction de qui que ce soit, pas même celle des contraintes légères d'un département universitaire ou d'un institut de science. Tout artiste ou romancier le comprendrait - certains d'entre nous ne produisent pas le meilleur d'eux-mêmes lorsqu'ils sont dirigés. » Le refus du dirigisme institutionnel rime avec la possibilité de vivre «la science » comme une activité créative avant tout. «Rares sont les individus créatifs dans les institutions et les universités ", sanctionne-t-il. (Lovelock, 2001, p. 2)

Lovelock ne s'arrête pas à cette critique artiste commune dans les milieux de l'entrepreneuriat post-soixante-huitards. Tout en adoptant des pratiques d'entrepreneur et en revendiquant la plupart des prédicats communément associés à l'ethos 1'entrepreneurial (comme le fait d'être créatif), il rêve le scientifique en artiste à la manière des Romantiques, comme «menant une vie solitaire ». Cela rime pour lui avec le fait de «travailler souvent à la maison ». Ainsi, à l'aide de ses petits chromatographes, il réalise des mesures décisives dans le laboratoire qu'il a établi dans le garage de sa résidentielle principale à Bowerchalke, ou dans sa résidence secondaire d'Adrigole ( $c f$. infra).

Toutefois, ces activités domestiques ne sont nullement suffisantes pour valider l'épithète d'«indépendant» que Lovelock s'accole. Ce n'est pas un hasard si l'imagerie du "personnage hors du monde » et "perdu dans les systèmes de pensée abstraits »- dont Lovelock se drapera fréquemment dans ses écrits à partir de son premier Gä̈a (1979a) - est traditionnellement associée, outre à l'artiste-philosophe romantique, à la figure ancienne du savant et à «la recherche académique faite dans une tour d'ivoire"; et, jamais, à l'entrepreneur scientifique (Boutillier, 2003, pp. 79-80). L'un des enjeux principaux des sections suivantes sera précisément de proposer un contre-récit décrivant Lovelock, non comme "un scientifique indépendant», mais comme "un scientifique entrepreneur» construisant et pérennisant socialement son double statut d'entrepreneur et de scientifique. ${ }^{2}$

\section{La critique de la culture entrepreneuriale et de la législation des brevets du Royaume- Uni}

Lovelock n'emploie jamais le qualificatif «entrepreneur» pour se désigner dans son autobiographie, mais il défend ce statut. Il lutte, argue-t-il, contre une désaffection pour l'entrepreneur profondément enracinée dans la société britannique, qui reprocherait à cette figure de ne pas emprunter « les chemins normaux de la promotion professionnelle » compris, à tort selon Lovelock, comme des voies plus méritoires. La tirade se poursuit avec deux revendications récurrentes chez Lovelock, qu'il associe volontiers : une exhortation faite à la nation britannique de se révolter contre sa stagnation voire son déclin, avec comme contremodèle vertueux les Etats-Unis voire les pays d'Europe continentale ; son adhésion à un modèle socioéconomique libéral, dont la logique a vocation à percoler jusque dans les métiers

\footnotetext{
${ }^{2}$ Ce faisant, nous laissons de côté un point important dans cet article : si Lovelock se considère et se décrit comme scientifique indépendant, ce n'est pas uniquement pour prévenir les potentielles accusations de conflits d'intérêt avec des groupes privés, ni non plus seulement pour se mettre en valeur face à ses collègues restant emprisonnés dans une routine académique - deux points qui sont discutés ici -, c'est aussi en partie parce que Lovelock se rêve savant et inventeur tels que décrits dans les histoires mythifiées des savants du XIXème siècle (et il n'hésite pas à suggérer des comparaisons entre Maxwell ou Darwin et lui).
} 
de la recherche publique (Lovelock, 2001, pp. 75 \& 144-145). En fait, Lovelock reconduit dans les grandes lignes le "récit classique" que de nombreux acteurs - politiciens, mais aussi universitaires - ont fait pour expliquer le retard compétitif de l'industrie et de la recherche britanniques, voire la fuite des cerveaux outre-Atlantique, dans les années 1960-70. ${ }^{3}$

Lovelock s'attarde évidemment sur les politiques de recherche. Si les États-Unis sont devenus pour lui un modèle et une terre d'accueil, ce n'est pas parce que "c'est là qu'est l'argent", mais plus vraisemblablement parce que s'y épanouit précocement une culture entrepreneuriale de l'invention mêlant recherches publique et privée. La question des brevets est longuement discutée par Lovelock. L'évocation du NIMR est ainsi l'occasion de pourfendre la législation des brevets et la réticence de la recherche publique à déposer des brevets dans les années 1940-60. En 1944, Lovelock se voit contraint de publier dans Nature deux publications qu'il qualifie de "'patent-blocking' », empêchant que ses découvertes - des désinfectants bactéricides - ne soient brevetées par un autre (Lovelock, 2001, p. 75). Il faudra attendre la fin de la guerre pour que les brevets d'inventions médicales publiques soient autorisés, entérinant un changement d'attitude nationale sur le sujet (Keith, 1981, pp. 102-103). En outre, si Lovelock se réjouit du fait qu'en 1956 le MRC ait déposé immédiatement un brevet pour son détecteur à ionisation d'argon - en conséquence de quoi, écrit-il, « nous fûmes libres de parlementer avec les manufacturiers d'instruments, qui seraient tenus de nous verser des redevances (royalties) »-, c'est pour mieux dénoncer la singularité d'une telle initiative " en Grande-Bretagne, $\{\ldots\}$ personne ne prend jamais la peine de faire breveter les idées » (Lovelock, 2010 ; Lovelock, 2001, p. 178).

D'après Lovelock, la récompense aménagée pour les inventeurs de la recherche publique jouerait un rôle décisif d'entraînement de la $R \& D$ nationale. Mais, lorsqu'il en vient à écrire qu' « il y a peu de bénéfices directs pour un fonctionnaire \{britannique\} à faire breveter une idée, puisqu' \{il\} n'a rien à y gagner » (Lovelock, 2001, p. 178), l'assertion est hyperbolique. ${ }^{4}$ En outre, Lovelock n'est guère objectif quand il dénigre spécifiquement les pratiques en vigueur dans son pays (et qu'il voit perdurer bien au-delà des décennies 1950-60). Certes, dans les années 1950, la protection et surtout la monétarisation de la propriété intellectuelle demeurent des pratiques négligées dans la recherche publique britannique (y compris au sein du MRC). Mais, elle l'est dans l'ensemble des pays industrialisés, et le demeure au cours des deux décennies suivantes. ${ }^{5} \mathrm{Y}$ compris aux États-Unis où la recherche publique se montre

\footnotetext{
${ }^{3}$ Ce récit peut être synthétisé comme suit : au début du XX ${ }^{\text {ème }}$ siècle, l'industrie britannique a été distanciée par ses homologues allemands et états-uniens. Puis, le Royaume-Uni est resté à la traine derrière les autres pays européens pendant «l'âge d'or» de croissance économique entre 1950 et 1973. Enfin, le pays a rattrapé peu à peu son retard à partir des années 1980, grâce une croissance de l'investissement dans le développement technologique alliée à une meilleure productivité industrielle, grâce à des transformations en matière de management de l'innovation et la recherche (dont une valorisation marchande accrue de la recherche publique plus de brevets déposés, notamment), et grâce à un repositionnement sur le marché international (Nicholas, 2014, pp. 2-4).

${ }^{4}$ Jusqu'au milieu des années 1980, le dépôt de brevets apporte de faibles revenus aux inventeurs de la recherche publique britannique - mais non : «nuls », comme le laisse entendre Lovelock! La situation évolue ensuite, explique Soraya de Chadarevian, puisque, en plus des revenus de royalties touchés par leur institution scientifique, les inventeurs peuvent individuellement aspirer à gagner plusieurs dizaines de milliers de livres pour une invention, contre moins de $£ 1,000$ jusqu'alors. Si « la Grande-Bretagne n'était pas dans une « culture du brevet » dans les années 1970 », elle y est rentrée sous le règne de Margaret Thatcher (Chadarevian, 2011, pp. $622-624 \& 601)$.

${ }^{5}$ Aux États-Unis, l'une des motivations explicites premières de l'adoption de l'incontournable 'Bayh-Dole Act' de 1980 sera précisément l'augmentation de la proportion des licences industrielles d'exploitation reliées aux brevets de recherche publique. Les disparités demeureront importantes d'une université à l'autre, avec aux extrémités du spectre l'université « de type tour d'ivoire » Harvard et le MIT comme champion du « capitalisme de campus ». (Greenberg, 2007, p. 63).
} 
parfois ingrate avec ses inventeurs, comme le père de l'ECD lui-même l'apprend à ses dépens. ${ }^{6}$

En définitive, conclut Lovelock, il aurait contracté à vie le virus de la recherche publique britannique des années 1950-60, c'est-à-dire gardé l'attitude qu'il avait au MRC, où déposer des brevets et négocier des royalties étaient des pratiques culturelles rares. Or, si Lovelock n'a jamais dégagé des rentes importantes grâce à ses brevets, il a su les valoriser d'une autre manière.

\section{VALORISER SES INSTRUMENTS : LES RACINES ET LE CEUR DE L'ENTREPRENEURIAT LOVELOCKIEN}

Pour s'établir comme entrepreneur après avoir quitté le NIMR au tournant des années 1960, Lovelock valorise sa capacité à déposer des brevets pour le compte de ses clients et employeurs, crée des entreprises et se constitue un portefeuille de nombreux clients et employeurs dans la durée. ${ }^{7}$

\section{Valoriser ses talents d'ingénieur et sa capacité à déposer des brevets}

Lovelock n'a pas cherché à générer des revenus directement grâce à ses brevets (par revente ou royalties). D'abord, il explique que « de nombreuses personnes de Mill Hill généraient une propriété intellectuelle de valeur ». Mais, bien que très courtisés à l'extérieur (Lovelock affirme qu'il a passé «une grande partie de sa dernière année à Mill Hill à repousser les compagnies d'instruments qui voulaient \{son\} expertise sur la chromatographie en phase gazeuse »), les gens de Mill Hill n'étaient pas tentés de céder aux sirènes de l'industrie car ils avaient « un fort sentiment de loyauté envers l'Institut et \{étaient\} bien payés et bien traités ». Plus : ils se pliaient à leur «devoir de quasi-fonctionnaire de conseiller gratuitement les firmes qui voulaient mettre \{leurs\} inventions sur le marché, en particulier celles qui utilisaient \{leurs\} brevets en vertu d'un accord de licence ». Pour accomplir son « devoir », Lovelock conseillera ainsi la compagnie, WG Pye, qui avait acheté une licence pour son détecteur à ionisation d'argon (Lovelock, 2001, pp. 139-140).

Il est bien l'inventeur de nombreux brevets, mais également le détenteur propre ou le codétenteur de certains d'entre eux - lorsqu'il affirme n'avoir « breveté à titre personnel qu'une

\footnotetext{
${ }^{6}$ Lors d'un séjour « sabbatique » à l'Université de Yale en 1958, relate Lovelock, son 'Department of Internal Medicine' lui conseille de déposer un brevet pour son ECD. Il accepte un partage au tiers des recettes entre l'Université, l'agence de brevets et lui. Le brevet paraît au journal officiel en 1964. Le département le contraint alors à accepter un nouvel accord qui « attribue le brevet au gouvernement états-unien ». L'inventeur maugrée quelque peu mais cède rapidement. Il «pens $\{\mathrm{e}\}$ alors que l'ECD \{est\} une invention mineure et qu'il \{est\} improbable qu'elle vaille beaucoup ». En fait, l'ECD connaîtra une riche postérité dans la recherche publique et privée. Or, si son nom figure sur le brevet de l'ECD, assure Lovelock, il n'aurait jamais touché de royalties pour cette invention (Lovelock, 2001, pp. 196-197).

${ }^{7}$ Dans les années 1980-90, deux approches s'opposaient dans la littérature sur l'entrepreneur. La première, «l'approche par les traits de personnalité, également appelée approche psychologique », est au fond celle privilégiée dans la section «Le récit lovelockien en question». On y voit apparaître en filigrane les «quatre caractéristiques essentielles » de « la personnalité kaléidoscopique » de l'entrepreneur selon cette approche : «le besoin de réalisation de soi »; « le lieu de contrôle interne » (« la capacité de l'entrepreneur à considérer que ce qui lui arrive dépend surtout de lui-même »); « le besoin de pouvoir » (« volonté affirmée de tout contrôler pour mieux maîtriser les évènements ») ; « la propension à prendre des risques »- les deux premières étant clairement plus affirmées chez Lovelock. La seconde approche, à laquelle s'affilient cette section et la suivante, est «l'approche par les comportements ». Elle focalise la recherche, non sur ce qu'est l'entrepreneur mais sur ce qu'il fait, et en particulier sur «comment se comporte l'entrepreneur dans son acte de création et de développement d'entreprise ». (Messeghem \& Sammut, 2011, pp. 50-59)
} 
seule de $\{$ s $\}$ es inventions » (Lovelock, 2001, p. 190), ce n'est donc pas exact. ${ }^{8}$ Mais il a délégué les droits et les royalties de nombreux brevets aux institutions et entreprises pour lesquelles il travaillait (Hewlett-Packard (HP), NASA, Shell). Déposer des brevets lui a permis de valoriser sur le marché des biens et services ses talents d'inventeur et de développeur d'instruments d'une autre manière.

Lovelock a, premièrement, mis en avant un savoir-faire et une veille technologique sur des instruments existants ou qu'il a inventés. Il offre ainsi une description de sa première expérience commerciale de promotion de brevets en 1962 : «Posséder le brevet d'une invention est comme avoir la graine d'un arbre fruitier. Tu ne peux pas cueillir le fruit avant d'avoir planté la graine, de t'être occupé de sa croissance, et à moins que tu n'aies eu la sagesse d'attendre que le fruit soit mûr et prêt à être cueilli ». Planter la graine, c'est passer un accord avec $F$ and M Scientific, une start-up qui lance son activité en construisant des chromatographes en phase gazeuse (et qui sera ensuite rachetée par HP), afin qu'elle réalise une première version commercialisable de l'ECD. S'occuper de son arbre jusqu'à ce qu'il aboutisse à des fruits, cela signifie que Lovelock « $\mathrm{d}\{$ oit $\}$ visiter la firme $\{F$ and $M\}$ deux fois par an pour discuter des problèmes et des prochains développements ». Cette « routine de visites » durera trente-deux ans (Lovelock, 2001, pp. 180-181). Le choix de l'industrie concessionnaire du brevet se prolonge par le suivi des développements technologiques au sein de cette industrie. Deuxièmement, Lovelock a valorisé son talent d'ingénieur susceptible de réaliser de nouvelles inventions, avec des brevets et donc des retombées à la clef pour ses employeurs potentiels.

\section{Créer des entreprises}

Afin de s'établir comme entrepreneur, Lovelock créé des entreprises. Il fonde sa première entreprise Ionics Research en 1962 avec Albert Zlatkis, professeur de chimie de l'Université de Houston. Il explique que, "en opérant comme une entreprise, il \{leur\} fut \{désormais\} possible d'offrir un conseil et de fournir des prototypes de détecteurs à n'importe quelle firme de l'industrie des instruments qui en avait besoin. » (Lovelock, 2001, p. 153). En 1965, Lovelock quitte officiellement Ionics, mais gardera des liens avec elle. ${ }^{9}$

Lovelock crée ensuite plusieurs petites entreprises, qui couvrent de nouveaux usages. En 1964, il fonde sa propre (et principale) entreprise Brazzos Limited. Elle lui sert à passer des commandes auprès de fournisseurs (d'instruments, de substances chimiques et radioactives, etc.), à facturer ses clients et à signer de multiples contrats de consultance. James Lovelock, sa femme Hélène, son fils Andrew et sa fille Christine en sont les quatre directeurs et membres uniques. Christine, dans les années 1970, et Andrew, dans les années 1980, travaillent épisodiquement avec leur père sur des mesures et l'élaboration d'instruments, respectivement.

En 1969, Lovelock devient vice-président de la société INSCON (International Science Consultants) initialement basée à Houston (Texas). INSCON propose des études conjointes avec Ionics et, parmi les contrats obtenus ou pressentis par INSCON, on retrouve les clients et employeurs de Lovelock (e.g. JPL, la NOAA, Panametrics, l'EPA, DuPont, Pye, Shell, HP,

\footnotetext{
${ }^{8}$ Il est en revanche possible que Lovelock n'ait vendu qu'un seul brevet. Nous avons retrouvé une seule transaction de ce type : la vente du brevet sur la 'Detection of trace gases utilizing an electron capture detector' à Dow Chemical en 1976 (pour 65000 \$) (Archives de Lovelock).

${ }^{9} \mathrm{Il}$ sera par exemple associé à une proposition de recherche déposée auprès de l'armée américaine par Ionics le 20 avril 1971 'Ultrasensitive ionization detection systems for trace analysis of atmospheric constituents' (Archives de Lovelock).
} 
des sous-traitants de la NASA comme Perkin-Elmer, le Département de la Défense des ÉtatsUnis $)^{10}$

\section{Alimenter un portefeuille de clients et d'employeurs constitué d'entreprises privées et d'institutions publiques solidement implantées}

Dressons la liste des principaux clients et employeurs de Lovelock. Ce dernier fournit des prestations à des grandes entreprises, comme HP (de 1964 à 1994), et surtout à des entreprises chimiques et pétrolières. Sa consultance pour Shell s'étale au moins de 1964 à 1984 - avec quelques années de prise de distance entre 1977 et 1980 liées à des problèmes de santé pour Lovelock et à une surcharge de travail engendrée par son implication dans la question de l'ozone ( $c f$. lettre de Lovelock à Rose 1977.09.19 \& lettre de Yeo à Lovelock 1980.10.09; Archives de Lovelock). Certaines de ses recherches, à partir du milieu des années 1970, sont financées par 1'US Chemical Manufacturers Association (CMA), un lobby promouvant les intérêts d'industries chimiques, d'autres par l'Imperial Chemical Industries (ICI) britannique. Il est aussi à plusieurs reprises en contact étroit avec DuPont. ${ }^{11}$

Les consultances ne sont pas seulement réalisées pour des entreprises, mais également pour des grandes institutions publiques: les services secrets britanniques (le MI5, pour qui il travaille pendant près de quarante ans, jusqu'à l'âge de 80 ans au moins) ${ }^{12}$ et les institutions scientifiques majeures des sciences de la Terre et de l'environnement. Outre ses travaux pour le JPL de la NASA (de 1961 à 1973), Lovelock obtient dans les années 1970 au moins un contrat important (de $100000 \$$ ) auprès de la NOAA (National Oceanic and Atmospheric Administration, l'agence américaine finançant des recherches en climatologie et océanographie). Lester Machta, directeur de la NOAA Air Resources Laboratory et ami de Lovelock, estime que, derrière les mesures de David Keeling sur l'augmentation du $\mathrm{CO}_{2}$ dans l'atmosphère dans la seconde moitié du XXème siècle, les travaux de Lovelock ont été les plus marquants pour la NOAA (parmi les travaux réalisés par des acteurs n'étant pas affiliés à l'institution). ${ }^{13}$

Les modalités des contrats que Lovelock signent dépendent du contexte. Tantôt il bénéficie d'une rémunération annuelle sur la base d'un contrat définissant à l'avance le travail qu'il doit effectuer - c'est le cas par exemple chez Shell dans les années 1960 (ses contrats prévoient des revenus annuels compris entre 100 et $2000 £)^{14}$ et chez HP dans les années 1980 (par exemple, le contrat signé en 1989 pour les trois années à venir prévoit 32 000\$/an; archives de Lovelock). Tantôt Lovelock facture ses services à la journée. De tels exemples abondent dans ses archives. Par exemple, au début des années 1970, il facture ses services au JPL $100 \$$ la journée ; le 29 mars 1982, il facture 1050\$ à DuPont pour 3 jours de consultance entre janvier et mars 1982 sur une « réflexion sur des méthodes indirectes permettant de détecter les variations d'UV solaires ". ${ }^{15}$ Tantôt enfin, il propose des devis à la tâche, pour une mission incluant les salaires et achats de matériels sans référence au temps de travail - ainsi, un devis de Brazzos à la NOAA en 1980 propose la livraison d'un prototype pour effectuer des mesures de perfluorocarbures (pour $40000 \$$ ).

\footnotetext{
${ }^{10} \mathrm{Cf}$. le compte rendu INSCON du 24 février 1971 (Archives de Lovelock).

${ }^{11}$ Entre le début des années 1970 et le début des années 1980, Lovelock échange très régulièrement, par lettres, avec DuPont (Frank Bower et Ray MacCarthy notamment), propose ses services de consultant, consulte ces derniers au sujet de ses travaux pour la CMA, etc.

${ }^{12}$ L'un de ses proches collaborateurs Andrew Watson suggère qu'il y aurait touché au moins la moitié de ses revenus en carrière (Discussion avec Andrew Watson réalisée par S. Dutreuil, 28.02.2018).

${ }^{13}$ [Lester Machta, entretien avec Weart et Elliott, AV, 1991]

${ }^{14}$ En 1969, Rothschild propose de changer les contrats pour payer Lovelock à la journée, $50 £$ (lettre à Lovelock du 17.06.1968).

${ }^{15}$ Facture du 29 mars 1982, archives de Lovelock.
} 
Lovelock choisit donc une trajectoire hybride, qui combine des contrats de recherche publics et privés, et qui fait une place à la fois à une activité de bricoleur et à la recherche théorique ( $c f$. ses travaux sur Gaïa, infra). Aux yeux de Lovelock, partir de Mill Hill pour embrasser une carrière d'entrepreneur, c'est refuser que sa vie ne "suive une trajectoire prédestinée ». C'est aussi fuir une carrière routinière (Lovelock, 2001, p. 139). Ce n'est donc pas pour retomber dans une autre routine, celle du simple usage-raffinement de ses instruments, comme le lui proposent des industriels au tournant des années 1960 (Lovelock, 2010 \& Lovelock, 2001, p. 140). En fait, Lovelock trouve une réelle stimulation intellectuelle dans le développement d'instruments, dont il déplore qu'il soit dénigré - à l'inverse, selon lui, des théories et des œuvres d'art (Lovelock, 2001, pp. 186-191). Mais, "construire, tester et vendre un produit \{i.e. un instrument $\}$ bien fait $\{\ldots\}$ est un travail à plein temps »; or, il veut aussi « dévouer $\{\mathrm{s}\}$ on temps à la science » (Lovelock, 2001, p. 5), c'est-à-dire faire une place à la fois à une activité de bricoleur et à la recherche théorique.

Reste que les instruments de chromatographie jouent deux rôles décisifs dans sa carrière. D'un côté, ils lui assurent une forme d'indépendance. Par leur caractère peu onéreux et aisément transportable, ils lui permettent en effet de travailler seul (ou presque seul), au moment du plein essor des «big sciences » de l'environnement global dont la tendance va à rebours d'une science solitaire (e.g. Conway, 2008, Edwards, 2010) À travers les mesures qu'il effectue, Lovelock garde ainsi une main locale sur l'environnement global. Et, si « les ventes d'instruments \{constituent\} une source peu fiable de revenus ", le développement d'instruments lui apporte des revenus qu'il juge confortables dès le début de son activité entrepreneuriale. Les contrats qu'il signe via 'Brazzos Limited' lui sont même « tous offerts, $\{$ il $\}$ ne les cherch $\{$ e $\}$ jamais », rapporte-il (Lovelock, 2001, pp. 5 \& 283). En outre, tout en lui assurant des formes d' " indépendance ", ses instruments jouent en même temps un deuxième rôle de mise en réseau avec des acteurs de l'industrie et de la recherche publique : ils sont sa marque de fabrique qui justifie l'obtention de la majorité de ses contrats de consultance et assurent la prospérité de l'entrepreneuriat de Lovelock pendant toute sa carrière. ${ }^{16}$

Lovelock diversifie ses activités de consultance dès le milieu des années 1960 en ajoutant des activités de conseil qui relèvent d'une expertise plus scientifique que technique : il devient bientôt une figure que l'on consulte au sujet des pollutions environnementales... des questions vers lesquelles l'ont, là encore, guidé ses instruments.

\section{ACTIVITES CONCRETES}

Les activités concrètes de Lovelock dans les années 1960 et 1970 se répartissent en quatre pôles: l'élaboration d'instruments, la mise au point de techniques chimiques et la veille instrumentale ; les mesures ; la rédaction de rapports et de notes de synthèse ; l'élaboration de l'hypothèse Gaïa. Nous les passons en revue, en commençant par les deux premières, qui occupent la plus grande place dans l'emploi du temps de Lovelock.

\footnotetext{
${ }^{16}$ Il n'est pas aisé d'avoir une idée précise des revenus dégagés par Lovelock au cours des années 1960 et 1970 , compte tenu du caractère épars des documents financiers dans ses archives et de la diversité de ses clients et employeurs. Nous travaillons sur cette question pour une publication ultérieure. Nous pouvons par contre rapporter des exemples de montants que Lovelock dit avoir touchés à ses débuts en 1961 : « un total de plus de $3500 £$ annuelles » émanant de la NASA et de Shell (soit, « juste ce qu'il fallait pour débuter un travail de scientifique indépendant » à l'époque, estime-t-il), auquel s'ajoute, immédiatement à son arrivée aux Etats-Unis, « un salaire de rêve de $20000 \$$ par an » provenant du Lipid research center de l'Université de Houston (Lovelock, 2001, p. $160 \& 145$ ).
} 


\section{Élaboration d'instruments, mise au point de techniques chimiques et veille technologique}

L'activité principale de Lovelock consiste à élaborer des instruments et à faire de la veille technologique. Le cœur de métier de Lovelock relève donc d'un savoir-faire. S'il est enthousiasmé à l'idée de participer, avec la NASA, aux réflexions théoriques portant sur les critères utilisés pour détecter de la vie sur d'autres planètes (qui joueront un rôle important dans l'élaboration de l'hypothèse Gaïa), l'activité pour laquelle il est originellement employé comme consultant au JPL, et pour laquelle il reste consultant pendant plus de dix ans, est bien l'élaboration de chromatographes. L'essentiel de la consultance de Lovelock auprès de HP consiste à faire de la veille technologique sur des chromatographes et l'ECD. La CMA finance les travaux de Lovelock pour transformer l'ECD en un instrument de mesure absolue, ainsi que pour élaborer et calibrer des chromatographes qui équipent des stations de mesure en Afrique du Sud (Lettre de Graeme Milne à Lovelock, 4.08.1976 et Contrat avec la CMA No 75-76, 1976, archives de Lovelock).

Ce sont à nouveau ses aptitudes à élaborer des techniques de mesures de composés chimiques qui sont recherchées par les services de renseignement, pour au moins deux activités : la détection d'explosifs; la mise au point d'un système permettant de tracer une personne cible (reposant sur un marquage chimique de la personne). ${ }^{17}$ À Shell, Lovelock est sollicité sur des questions instrumentales, par exemple lorsqu'il doit mettre au point un système permettant de détecter une fuite dans un pipeline, ou une méthode permettant d'estimer la concentration en dioxyde de soufre dans l'atmosphère et l'origine des masses d'air le contenant. Mais, pas seulement : il est également appelé (et parfois propose lui-même ses services) plus largement comme un ingénieur auquel on soumet des problèmes techniques : métal à utiliser dans des batteries, extraction d'or en suspension dans une rivière ${ }^{18}$, utilisations possibles du méthane, modalités de transport de gaz naturel par voie maritime ${ }^{19}$, possibilité d'extraire de l'énergie à partir de phénomènes d'osmose, etc.

\section{Mesures et pollution}

Lovelock ne se contente pas de concevoir et d'améliorer des instruments : il les utilise pour effectuer des mesures importantes de chimie de l'atmosphère et des océans. Il effectue des mesures régulières et dans la longue durée à ses deux domiciles de Bowerchalke et Adrigole. Ses archives sont emplies de tableaux de mesures de turbidité de l'atmosphère, de température et de direction du vent, qui commencent à la fin des années 1960 et s'étirent dans toute la décennie 1970. Il effectue en outre des campagnes de mesure à bord de navires océanographiques (le Shackleton en 1971-1972 et le Meteor en 1973), et, ponctuellement, à bord d'un avion évoluant dans la stratosphère. Ces campagnes lui permettent d'effectuer les premières mesures globales de CFC dans l'atmosphère (Lovelock et al., 1973), de documenter l'émission de sulfure de diméthyle (DMS) (Lovelock et al., 1972) et d'établir une répartition des nitrates de peroxyacétyle (Lovelock et Penkett, 1974). Pour réaliser ses mesures, Lovelock est aidé initialement d'un doctorant, Robert Maggs. Et, à Adrigole, Lovelock délègue la tâche des mesures à un voisin qu'il forme pour l'occasion.

\footnotetext{
${ }^{17}$ Lovelock décrit une partie de ses activités pour les services de renseignement dans son autobiographie. D'autres éléments nous ont été apportés lors de discussions avec Andrew Watson. Certains rapports dans les archives de Lovelock apportent également des précisions utiles sur le genre d'activités menées par Lovelock dans ce cadre, comme le rapport déposé auprès de l'armée américaine par Ionics le 20 avril 1971, intitulé 'Ultrasensitive ionization detection systems for trace analysis of atmospheric constituents'.

${ }^{18}$ Note « Discussions at Sittingbourne », 23.11.1965, archives de Lovelock.

${ }^{19}$ Lettre de Tony Fish à Lovelock, 9.11.1967.
} 
Dans son autobiographie, Lovelock raconte que les premières mesures sont faites à son initiative, alors qu'il s'inquiétait de pollutions de l'air inhabituelles dans son environnement (Lovelock, 2001 p. 204). En réalité, plusieurs éléments indiquent que ces mesures initiées au tournant des années 1960-70, toutes liées à des problèmes de pollution résultant de la combustion d'énergies fossiles, sont encouragées entre autres par Shell. ${ }^{20}$ Outre des mesures précises, Lovelock fournit des échantillons d'air à Shell, qui sont ensuite analysés par d'autres pour les besoins d'études portant sur la répartition des insecticides produits par Shell, comme l'aldrine ou la dieldrine. ${ }^{21}$ Enfin, à partir du milieu des années 1970, alors que les CFC viennent d'être mis en cause comme possibles destructeurs d'ozone, la CMA finance les mesures de différents halocarbures menées à Adrigole par Lovelock dans le cadre du programme Atmospheric Lifetime Experiment (e.g. Prinn et al., 1983). ${ }^{22}$

\section{Rédaction de rapports et de notes de synthèse}

Lovelock est également sollicité par ses clients et employeurs en tant qu'expert scientifique (par opposition à expert technique), pour rédiger des notes de synthèse sur une question précise ou des notes prospectives. Dès les années 1960, Shell est par exemple très intéressé d'entendre Lovelock sur la question émergente du changement climatique. À partir de 1966, il rédige ainsi plusieurs notes de synthèse de quelques pages, dont la première est 'Combustion of fossil fuel: large scale atmospheric effects' (9.06.1966). Pour Shell encore, à la demande de Rothschild, Lovelock rédige une note prospective sur l'état du monde en 2000 et sur la place que pourrait occuper Shell en des temps troublés par l'augmentation de la démographie et l'émergence de problèmes environnementaux globaux ('Some thoughts on the year 2000', novembre 1966). Un autre rapport propose une «perspective systémique sur l'activité minière $»(1969)$.

De manière plus large, certaines des entreprises qui emploient Lovelock voient dans ce scientifique entrepreneur, non pas seulement l'ingénieur-chimiste et l'inventeur d'instruments hors pair, mais également le chercheur universitaire et académique inséré dans plusieurs réseaux disciplinaires allant de la chimie aux sciences de l'environnement et de la Terre en passant par la biologie. Pour prendre un autre exemple, plus tardif, Lovelock participe en 1985 à une conférence organisée à Sao Paulo par les Nations Unies sur la place des forêts tropicales humides en climatologie; en amont de la conférence, Colin Quinn (Shell) lui demande de rédiger une note sur son contenu avec en vue toutes les questions pouvant affecter une entreprise impliquée dans les activités forestières au Brésil. ${ }^{23}$

\section{Élaboration de l'hypothèse Gaïa}

La dernière activité de Lovelock consiste à élaborer l'hypothèse Gaïa. Sa première formulation écrite est la note prospective pour Shell de novembre 1966 (Dutreuil, 2016). Elle a ensuite été publiée par Lovelock à la fin des années 1960, et activement développée dans

\footnotetext{
${ }^{20}$ Parmi ces éléments, on trouve des remerciements en fin d'articles publiés par Lovelock (e.g. Lovelock et al., 1972, p. 453), mais aussi des documents non publiés écrits par Lovelock pour Shell (e.g. « The source and role of minor atmospheric components », rédigé entre 1971 et 1973), ou des lettres échangées entre Lovelock et Shell (e.g. lettre à Lovelock du 13.12.1973).

${ }^{21}$ Voir le rapport confidentiel 'The concentration of aldrin, dieldrin and their photoisomers in air samples taken during July, 1973 in S.W. Ireland' (Shell research limited, London), conduite par D. Bennett (Archives de Lovelock).

${ }^{22}$ Les financements de la CMA ont aussi bénéficié à des chercheurs universitaires, ainsi que le rappelle souvent Lovelock pour tempérer les accusations de conflit d'intérêt.

${ }^{23}$ Lettre de Colin Quinn à Lovelock, 20.04.1983.
} 
une série d'articles dans les années 1970, pour la plupart co-écrits avec la microbiologiste américaine Lynn Margulis, puis dans un livre en son nom propre (Lovelock, 1979a).

Ces textes, évoquant une série de mécanismes précis liant les vivants à leur environnement géologique, sont formulés à un haut niveau de généralité et s'accompagnent de revendications méthodologiques et programmatiques pour les sciences de l'environnement et de la Terre. L'apport principal de Gaïa n'a pas été la formulation d'une " hypothèse » qu'il s'agirait de tester pour savoir si elle est vraie ou fausse (i.e. l'environnement global est-il, ou non, « bien régulé »?), mais bien plutôt une contribution ontologique qui a eu des implications épistémologiques importantes : Gaïa désigne un nouvel objet, le système complexe constitué de l'ensemble des vivants et de l'environnement physique, chimique et géologique avec lequel ils interagissent. La présentation de ce nouvel objet a présidé à la constitution des «sciences du système Terre » à partir des années 1980 et a, ce faisant, reconfiguré en profondeur les sciences de l'environnement et de la Terre : d'une part en mettant en lumière les interactions complexes qui existent entre les molécules chimiques de l'atmosphère, l'activité des vivants et la physique du climat, et d'autre part en soulignant explicitement les conséquences épistémologiques qui doivent être tirées pour les sciences et savoirs de l'environnement et de la Terre de la reconnaissance d'un nouvel objet ou d'une nouvelle perspective sur la Terre (Dutreuil, 2016).

Telles sont les principales activités de Lovelock au cours des décennies 1960-70: développement $d$ 'instruments et veille technologique, mesures atmosphériques et océanographiques, rédaction de notes et de rapports, écriture d'articles scientifiques. Une partie importante de ces activités était réalisée chez lui, dans son laboratoire. Là, il recevait également ses interlocuteurs (de Shell, de HP, des services de renseignement, des collaborateurs scientifiques, etc.). Pour le reste, il se déplaçait : en Angleterre dans les locaux de Shell à Sittingbourne ou à Thornton; dans les locaux des services de renseignement à Holton Heath; il effectuait également des tournées américaines de quelques semaines au cours desquelles il s'arrêtait au JPL, à Boulder où se trouvaient le NCAR (National Center for Atmospheric Research) et la NOAA, chez HP à Avondale, à Boston pour échanger avec Lynn Margulis, à Houston, ainsi que dans d'autres villes pour participer à des conférences.

Toutes ces activités ont permis à Lovelock de conserver à la fois un pied dans le monde des affaires, via ses divers contrats pour des entreprises privées, et un autre dans le monde académique, par des contrats au fil de l'eau, via ses affiliations académiques à l'Université de Houston (1961-64) puis de Reading (1967-89), ses participations à des congrès, ses publications dans des revues scientifiques (ses mesures atmosphériques dans Nature, ses travaux sur Gaïa dans des revues de géosciences, ses découvertes et améliorations d'instruments dans des journaux spécialisés de chimie analytique).

\section{GARDER UN PIED DANS LES MONDES ACADÉMIQUE ET INDUSTRIEL : SYNERGIE DES RÉSEAUX ET DES ACTIVITÉS DE LOVELOCK}

S'il est particulièrement intéressant de souligner cette double appartenance, c'est premièrement parce que, dans le cas de Lovelock, elle s'accompagne d'une singularité qui n'a pas été documentée à propos d'autres scientifiques entrepreneurs : en élaborant l'hypothèse Gaïa, Lovelock ambitionne une contribution théorique, spéculative ou programmatique de fond dans certaines disciplines. C'est, deuxièmement, parce que cette double appartenance est le lieu de synergies très importantes, que Lovelock met peu en avant. Nous montrons ici l'importance de ces synergies institutionnelles, puis soulignons le rôle de trois «éléments unificateurs » favorisant ces synergies et proposons quelques perspectives sur les éventuels conflits d'intérêt entre les activités de Lovelock. 


\section{Un réseau public-privé intriqué, avec des thématiques de recherche communes}

Lovelock est loin d'être le seul, y compris dans son entourage, à circuler entre la production de connaissances publiques et le conseil aux entreprises privées. Pendant ses années de consultance pour Shell, il retrouve ses anciens collègues de Mill Hill: John 'Kappa' Cornforth (Nobel de chimie 1975) et George Joseph Popják, recrutés par Rothschild en 1962. Peter Simmonds, son assistant de recherche à Mill Hill à la fin des années 1950, suit Lovelock dans ses différentes institutions : Université de Houston de 1961 à 1967 ; JPL à partir de 1967 ; services secrets à partir de la fin des années 1960. Il est également vice-président de INSCON depuis 1969, et reprend les rênes de l'entreprise qui est chargée de poursuivre des mesures de chimie atmosphérique à Mace Head en Irlande après la fermeture de la station d'Adrigole en 1978. Il n'est pas étonnant de retrouver des anciens collègues du MRC parmi ces entrepreneurs, puisque l'institution soutient ses chercheurs dans leurs démarches de prendre des consultances rémunérées par le privé (dès la fin des années 1960, elle les y incite officiellement $)^{24}$. Quant à Dian Hitchcock, philosophe à la NASA, elle n'a pas seulement coécrit avec Lovelock un article important sur la détection de la vie sur d'autres planètes (Hitchcock et Lovelock, 1967) ; elle s'est également révélée être une source utile de contacts en suggérant à Lovelock de présenter certaines de ses inventions aux services de renseignement américains (Lovelock, 2001, p. 169 sq.).

Les institutions pour lesquelles Lovelock travaille ne se situent jamais sur un seul et unique plan. Mill Hill, l'Université de Houston, le JPL (NASA) et l'Université de Reading, c'est-àdire les affiliations académiques principales de Lovelock dans les années 1950-60, ne sont pas des institutions académiques repliées sur la seule production académique de connaissances : elles sont pour Lovelock l'occasion de rencontres qui contribueront à inspirer, renforcer et élargir son réseau de consultant. Des liens complexes, d'appartenance multiple, lient ainsi les divers acteurs d'Ionics aussi bien à JPL qu'à l'Université de Houston et à Shell. De même, les entreprises privées se sont révélées constituer un appui important pour les travaux de Lovelock ensuite reconnus dans la sphère académique. Par exemple, Shell et la CMA financent des mesures qui donneront lieu à la publication de nombreux articles dans Nature, et Shell (et, plus tardivement, HP) ont soutenu et encouragé Lovelock dans son développement de l'hypothèse Gaïa. ${ }^{25}$ Lorsque Lovelock a besoin d'effectuer des mesures pour alimenter les débats sur l'ozone stratosphérique, il peut compter sur des contacts au Met Office, avec lequel il est en relation étroite depuis la fin des années 1960, et au Ministère de la Défense, qui lui obtient un avion (Lovelock, 2001, p. 217). Nous le voyons, Lovelock construit un réseau à cheval sur le public et le privé, qu'il parvient parfois à mobiliser de manière presque indifférente sur ses deux thématiques phares, la pollution atmosphérique et Gaïa.

Des synergies existent en outre entre ses activités au sein d'une même institution. Arrêtonsnous à titre d'exemple sur la relation longue et riche que Lovelock entretient avec le temple de la climatologie américaine, le NCAR, à partir du milieu des années 1960. Le NCAR est le client du concepteur d'instruments, à qui il passe commande de chromatographes en $1972 .{ }^{26} \mathrm{Il}$

\footnotetext{
${ }^{24} C f$. Soraya de Chadarevian explique que «depuis la fin des années 1960, un nombre de scientifiques du LMB toujours faible mais en progression avait aussi pris des consultances scientifiques payées dans diverses entreprises d'instruments, chimiques, pharmaceutiques, dont DuPont et Schering. Cette activité secondaire était autorisée par le MRC et accoutumait les scientifiques au monde des affaires » (Chadarevian, 2011, p. 607).

${ }^{25}$ Les soutiens les plus forts sont venus de Lord Rothschild et Sydney Epton. Le premier, Fellow of the Royal Society, a soumis une publication de Lovelock comme Proceeding of the Royal Society (Lovelock, 2001, p. 249). Le second a co-signé avec Lovelock un article fameux sur Gaïa (et la pollution), paru dans New Scientist.

${ }^{26} \mathrm{La}$ commande est passée le 10.03.1972 par le NCAR pour deux chromatographes d'une valeur de $1750 \$$ et 1250\$, archives de Lovelock.
} 
est aussi une source d'informations et de données brutes pour le scientifique Lovelock qui s'intéresse à des questions de pollution, de météorologie et de climatologie. ${ }^{27}$ Occasionnellement, le NCAR lui transmet également des échantillons d'air récoltés lors de campagne, qu'il analyse pour ses propres recherches. ${ }^{28}$ Ces interactions scientifiques sont soutenues et encouragées par Shell, qui s'intéresse de près aux réflexions scientifiques sur la pollution de l'air et le climat dès les années 1960, alors que ces sujets gagnent du terrain au sein de la communauté scientifique. ${ }^{29}$ Le contact et ami de Lovelock au NCAR, le chimiste James Lodge, est également éditeur de la revue Atmospheric Environment, dans laquelle Lovelock publie le premier texte portant le nom de «Gaïa » (Lovelock, 1972), mais aussi des textes sur le changement climatique (Lovelock, 1971). Le succès de Lovelock comme entrepreneur suscite d'ailleurs la vocation de Lodge qui lui demande des conseils pour s'établir comme indépendant. ${ }^{30}$ En 1975, Lovelock est convié à une conférence internationale décisive sur le climat, organisée par le directeur du NCAR Walter Orr Robert et l'anthropologue Margaret Mead. En 1985, pour les 25 ans du NCAR, Lovelock est invité par l'institution à donner une grande conférence pour impulser une vision nouvelle à la climatologie sur la base de ses travaux sur Gaïa. Ainsi, le NCAR joue vis-à-vis de Lovelock, tour à tour le rôle de client, de sources de données brutes, et d'appui institutionnel important pour faire rayonner Gaïa dans les sciences du climat.

En définitive, on ne saurait accepter la tonalité dominante du récit de Lovelock, d'après lequel tout se passe comme si ses travaux de consultant n'étaient qu'une simple source de revenus lui permettant ensuite, sur le temps qu'il lui reste, de développer ses propres recherches scientifiques. Contrairement à ce qu'affirme Lovelock, il ne consacre pas qu' "une partie faible de son temps $\{\ldots\}$ aux petits contrats de recherche et aux consultances », pas plus que ses travaux de consultance ne constituent uniquement des « travaux destinés à faire bouillir la marmite ('potboilers')» (Lovelock, 2001, p. 5). Au contraire, des complémentarités et des synergies existent de manière systématique entre ses différentes activités, sur les plans social et épistémologique.

\section{Trois éléments unificateurs : les instruments, Gaïa et les molécules}

Sur le plan épistémologique (et matériel), les synergies se cristallisent autour de trois éléments. Le premier est l'usage que Lovelock fait des chromatographes à partir du milieu des années 1960. Détecter une fuite de pipeline, suivre une masse d'air dans l'atmosphère ou une masse d'eau dans l'océan, ou encore détecter une molécule chimique pour suivre à la trace un espion ou un individu : toutes ces activités sont conduites avec le même instrument (l'ECD) (Lovelock, 2001, p. 176). ${ }^{31}$ De là le fait que nous soulignions, en début d'article, l'importance du talent de Lovelock à inventer des instruments détectant les molécules séparées par les chromatographes. C'est cette qualité qui lui permet de passer de manière fluide d'applications

\footnotetext{
${ }^{27}$ C'est ce dont témoignent par exemple les nombreux échanges épistolaires entre James Lodge et Lovelock qui remontent au moins au début des années 1970.

${ }^{28}$ Cf. par exemple lettre de Dieter Ehalt à Lovelock du 7 février 1972 (et la réponse de Lovelock le 14 février).

${ }^{29}$ Le 17 janvier 1967, Lovelock écrit à Rothschild qu'il essaiera de s'arrêter au NCAR lors de sa prochaine visite aux États-Unis pour avoir une vision plus complète et détaillée sur l'atmosphère actuelle, à la fin d'une lettre dans laquelle il est question de l'effet des aérosols sur l'atmosphère et du soufre sur la croissance des plantes. Puis, le 10 juillet de la même année, il lui écrit : « la meilleure manière de rester en contact avec le NCAR est par des visites assez fréquentes. J'ai une invitation à passer une semaine ici l'automne prochain et je peux inclure le NCAR comme un arrêt régulier de mes visites bi-annuelles aux USA. » (Archives de Lovelock).

${ }^{30}$ Lettre de Lodge à Lovelock, 15.07.1974, Archives de Lovelock.

${ }^{31}$ Pour une histoire des synergies existant entre l'essor des sciences de l'environnement et de la Terre et les activités militaires de la Guerre froide, voir par exemple : Doel, 2003 ; Turchetti \& Roberts 2014. Sur Lovelock en particulier, voir Grevsmühl, 2012.
} 
en biochimie médicale dans les années 1940-50 aux chromatographes pour le JPL et pour des industries chimiques et pétrolières au début des années 1960, et enfin à des mesures de pollutions environnementales générées par ces industries et documentées par ces dernières ainsi que par les grandes institutions des sciences de l'environnement et de la Terre (NERC, NOAA, NCAR, Met Office) à partir du milieu des années 1960. Sur ces questions de pollution, Lovelock se trouve sur la ligne de front. Comme nous l'avons montré ailleurs, ses diverses activités dans les années 1960 et 1970 le placent, soit au centre, soit comme témoin indirect et commentateur de la totalité des problèmes de pollution qui apparaissent et qui sont classiquement liés à l'émergence d'une conscience environnementale globalisée : le DDT décrié par Rachel Carson, produit par Shell puis mesuré dans l'environnement grâce à l'ECD par des chercheurs de Shell ; les CFC produits par DuPont et ICI, mesurés avec l'ECD par Lovelock, qui se retrouve impliqué dans l'affaire de la destruction de la couche d'ozone ; le plomb contenu dans l'essence des industries pétrolières, qui cause des problèmes sanitaires ; les pluies acides et le changement climatique, causés respectivement par le dioxyde de soufre et les gaz à effet de serre libérés lors de la combustion d'énergies fossiles; les nitrates de peroxyacétyles des smogs urbains (Dutreuil 2016, 2017).

Les synergies liées à une unité technique (les chromatographes et les détecteurs chimiques associés, en particulier l'ECD) sont redoublées par - et liées à - celles associées à un programme théorique : l'hypothèse Gaïa. Lovelock décrit volontiers Gaïa comme « un travail à temps partiel » (Lovelock, 2010). À le lire superficiellement, on peut avoir l'impression que l'élaboration de l'hypothèse Gaïa n'est que le fruit de réflexions spéculatives sur la vie ou la nature d'un Romantique retiré à la campagne. Or, ainsi que nous avons pu le développer ailleurs, Gaïa est un carrefour puissant des différentes facettes des activités de Lovelock : celles de consultant pour des entreprises privées mais aussi des institutions scientifiques comme la NOAA sur des questions de pollution de l'environnement global, puisque l'élaboration même de Gaïa s'ancre profondément dans ces réflexions sur la pollution et puisque Gaïa a en retour servi de cadre pour penser à nouveaux frais la pollution de l'environnement global ; celle de scientifique élaborant un programme de recherche nouveau pour refonder les sciences de la Terre, puisque Gaïa a proposé une nouvelle conception de la Terre ayant eu des effets profonds sur la restructuration des sciences de l'environnement et de la Terre; celle d'intellectuel et de scientifique se présentant auprès du grand public avec de nouvelles conceptions de la vie, de la Terre, de la nature et de la pollution (Dutreuil, 2016, 2017). ${ }^{32}$ C'est d'ailleurs précisément parce que Lovelock et Gaïa sont au carrefour de toutes ces questions qu'ils ont inspiré de nombreuses représentations nouvelles de la Terre et de la nature, occupant aujourd'hui une place décisive au sein des réflexions sur les changements globaux.

Enfin, en sus des instruments et de Gaïa, un autre facteur a opéré des synergies entre les différentes activités de Lovelock: les molécules chimiques. On pourrait ici rappeler

\footnotetext{
${ }^{32}$ Ce que nous voyons naître ici, ce sont deux autres facettes possibles de l'entrepreneuriat que Lovelock développera à partir des années 1980 (c'est-à-dire postérieurement à notre période d'étude) : une figure d'entrepreneur-intellectuel, qui communique des réflexions extérieures à son champ (des réflexions politiques, notamment) dans les médias et ses essais sur Gä̈a; une figure d'entrepreneur scientifique qui conçoit et promeut des programmes de recherche pour alimenter et réorienter son champ scientifique - le type d'entrepreneur scientifique que décrit Robert Friedman en prenant pour exemple le météorologiste Vilhelm Bjerknes (Friedman, 1989). Ce second rôle, Lovelock ne l'a que très marginalement endossé. Si Gaïa a bien mené à une transformation importante des sciences de la Terre avec la constitution des sciences du système Terre, et si Lovelock a certes écrit des articles programmatiques ambitionnant de telles transformations, il n'est pas l'acteur qui a activé les leviers institutionnels. Sur cette question, il faudrait par exemple souligner tout ce qui sépare l'éthos et les activités de Lovelock de ceux de Will Steffen, administrateur de la recherche ayant un rôle pivot au sein de l'IGBP, l'institution associée à la constitution des sciences du système Terre (Dutreuil, 2016, chapitres 8, 9 et 10$)$.
} 
l'importance des mesures de CFC réalisées par Lovelock et leur place décisive dans la guerre de l'ozone : outre des prix prestigieux récompensant le caractère pionnier de ces mesures, elles ont permis à Lovelock d'obtenir des financements de la CMA pour poursuivre ses mesures. Nous préférons nous arrêter ici sur le DMS. Dans son autobiographie, Lovelock «l'indépendant» se met en scène partant, les bottes au- pied, le chromatographe dans une main et un guide naturaliste d'identification des algues dans l'autre, réaliser une promenade et des mesures en bord de mer de son cottage d'Adrigole, lui permettant de remarquer que les algues comme Polysiphonia, émettent du DMS (Lovelock, 2001, p. 311). En 1972, Lovelock publie dans Nature les résultats de mesures effectuées dans l'Atlantique à bord du navire Shackleton et suggère que les quantités importantes de DMS émises par les algues pourraient constituer le flux principal, jusqu'alors non identifié, de soufre depuis l'océan vers l'atmosphère et les sols (Lovelock et al., 1972). Et en 1987, Lovelock et trois collègues suggèrent, dans Nature encore, que l'émission par les algues de DMS, ayant un rôle décisif dans la nucléation des nuages, pourrait contribuer à réguler le climat (Charlson et al., 1987). Dans ses ouvrages, Lovelock met donc en scène les mesures de DMS comme le fruit de son indépendance et participant à la naissance d'une science gaïenne réfléchissant aux grands cycles et équilibres de la nature. Or, à partir de la fin des années 1960, l'émission de dioxyde de soufre issue de la combustion d'énergies fossiles est considérée comme la cause principale des pluies acides. Alors que l'industrie britannique est directement mise en cause par la Scandinavie voisine, Lovelock en vient à tempérer cette incrimination compte tenu de l'existence de sources «naturelles » et «biologiques » de soufre dans l'atmosphère, via le DMS (e.g. Lovelock, 1995, p. 152-153). Suivre certaines molécules comme les CFC et le DMS (ou encore le méthane) permet donc, là encore, de mettre à jour le nœud gordien qui lie certaines de ses activités de consultant pour des entreprises privées et sa vie académique.

\section{Un homme « acheté par l'industrie »? Quelques perspectives sur les éventuels conflits d'intérêts traversant les activités de Lovelock}

Cela déplace la question de l'indépendance de Lovelock sur un autre plan : le fait que l'expertise environnementale de Lovelock pour des groupes privés entre en tension avec des travaux de groupes d'experts internationaux ancrés pour la plupart exclusivement dans la recherche publique pose la question du potentiel conflit entre les prises de position publiques de Lovelock et ses intérêts propres dans l'industrie. Lovelock s'est souvent défendu d'être un homme "acheté par l'industrie » pour ses positions au sujet de diverses controverses environnementales majeures, notamment celles sur les CFC et l'ozone dans le sillage de son témoignage comme expert devant le Congrès américain en $1974 .{ }^{33}$ Ces accusations à l'endroit de Lovelock, relativement rares à l'écrit, sont pourtant fréquentes dans les années $1970 .{ }^{34}$ Il est d'ailleurs intéressant de remarquer que c'est à cette même époque que Lovelock met en scène pour la première fois sa qualité de "scientifique indépendant», dans un article paru dans New Scientist (Lovelock, 1979b). Il est vrai que ses thèses sont plutôt confortables pour les industries chimiques.

Parce qu'elles minimisent l'implication des industries chimiques dans des pollutions environnementales, ces thèses pourraient être interprétées comme faisant système: un système idéologique sapant systématiquement les expertises sur les risques industriels sur la santé et l'environnement, à la manière des «marchands de doute » d'Oreskes \& Conway, 2010. Or, s'il est vrai que Gaïa est décrite dans les années 1970 comme fortement résiliente face à la grande majorité des perturbations produites par l'industrie, Lovelock peut devenir

\footnotetext{
${ }^{33}$ Sur la place particulière de Lovelock dans la controverse sur l'ozone, voir Briday, 2014. Sur les liens entre Gaïa et la pollution, voir Dutreuil, 2017.

34 Entretien avec Andrew Watson réalisé par Sébastien Dutreuil le 27.02.2018.
} 
très alarmiste au sujet des émissions modifiant le climat (Lovelock, 2006). ${ }^{35}$ En fait, les relations que Lovelock entretient avec l'industrie, d'une part, et les environnementalistes, d'autre part, sont complexes. Elles sont indissociables du scientisme que cultive le chimiste (et qui l'amène à être condescendant avec «les Verts"), mais aussi des reconfigurations épistémologiques, institutionnelles et politiques (avec la création de grands groupes d'expertise à la recherche de consensus scientifiques) qui s'opèrent entre les années 1960 et 1990. Les prises de position de Lovelock ne peuvent se réduire à quelques schémas trop simples et doivent faire l'objet d'une analyse détaillée, que nous proposerons dans un autre texte.

Pour conclure : plutôt que séparer les travaux universitaires des travaux de consultant dans le privé, comme le fait souvent Lovelock, nous avons montré au contraire la grande proximité et la grande synergie qui existent entre ces deux mondes. Nous aurions pu également signaler l'ingratitude de Lovelock vis-à-vis de la recherche publique: d'un côté, il pourfend son fonctionnement; de l'autre, il s'en nourrit et y cherche une légitimité. Insistons par ailleurs sur le fait que ce ne sont pas des contrats avec de petites start-ups qui constituent la terre ferme de la stratégie entrepreneuriale de Lovelock - contrairement à la stratégie d'autoentrepreneur qui se répand dans les nouvelles technologies (biotechs et informatique, notamment) au cours de la décennie 1970 -, mais des contrats avec de grandes institutions solidement installées dans le paysage de l'industrie ou de la recherche publique. Avec ce choix, Lovelock s'assure une stabilité financière et se donne un accès à ce qui se fait de mieux dans le domaine de la connaissance comme du développement technologique. Enfin, il comprend avant beaucoup d'autres que les enjeux liés aux pollutions de l'environnement global sont amenés à l'avenir à bouleverser la science et la politique.

\section{CONCLUSION}

Nous nous sommes focalisés dans ce texte, non sur les mutations institutionnelles de la recherche dans les années 1960-70 mais sur un scientifique individuel. Nous avons vu apparaître une figure précoce d'entrepreneur dans le milieu des sciences de l'environnement qui, issu de la recherche publique et gardant un pied dans celle-là, valorise sa capacité à inventer et déposer des brevets, et crée de petites entreprises dans lesquelles il raffine ses instruments et qui lui permettent de facturer des travaux de conseil variés. Des profils d'entrepreneurs scientifiques semblables existent déjà lorsqu'il se lance, et Lovelock fait des émules peu après ses débuts.

Lovelock n'est pas un entrepreneur « au sens traditionnel dans l'académie », de type Richard Feynman, qui «possède un talent éminent pour transformer une aptitude intellectuelle en réputation » au sein de son champ («talent» que, dans un cadre de compétition néolibérale, le « chercheur-entrepreneur » doit faire valoir sous la contrainte de techniques de gouvernement comme le benchmarking, les systèmes de classement, les grilles d'évaluation...) (Shapin, 2008, p. 220 ; Benninghoff, 2011). À l'inverse de son compère d'Ionics Al Zlatkis, Lovelock ne centre pas son activité sur le champ académique de la chromatographie. Il conserve constamment un pied dans la recherche publique et un autre dans la recherche privée, au sein

\footnotetext{
${ }^{35}$ Lovelock a modéré ses positions par la suite (Lovelock, 2009). Ses changements fréquents de position au sujet de l'importance du changement climatique ont donné lieu à de nombreuses inquiétudes et interprétations de la part de ses collègues climatologues et des ONG environnementales. Plutôt que dresser un historique des hésitations de Lovelock au sujet de la gravité des changements globaux, nous pensons plus utiles de souligner sa constance quant aux coupables désignés, qui sont toujours les mêmes depuis les années 1960: les comportements individuels et la surpopulation (Dutreuil, 2016, p. 657 sq.).
} 
desquelles il puise et produit des connaissances, et propose ses services. Toutefois, il ne colle pas non plus exactement au profil du nouveau scientifique entrepreneur décrit par Shapin. Si Lovelock fustige le dénigrement de l'entrepreneur qui a cours dans la société britannique, son projet est autre que la propagation de nouvelles technologies ou l'appât du gain (le "having fun, making money" des entrepreneurs shapiniens). Sa consultance est conçue comme devant contribuer à une quête de connaissances de pointe dans les deux unités thématiques qui deviennent rapidement ses préoccupations centrales : les pollutions atmosphériques et Gaïa.

Enfin, la mutation des pratiques universitaires market-oriented et de la protection de la propriété intellectuelle que décrit la littérature ${ }^{36}$ est plurielle. Lovelock ne s'inscrit pas dans celle que décrit spécifiquement Shapin. L'historien met au cœur de son récit les domaines des biotechs et de l'informatique. Il y observe que, à la fin des années 1970, de nombreux professeurs réorientent leur activité académique et créent leur propre entreprise pour produire un savoir et des instruments utiles à l'industrie, avec comme première visée explicite de générer des profits économiques (le 'spin-off syndrome'). Or, en quittant le milieu biomédical, Lovelock s'est éloigné de ces milieux où le " capitalisme académique » va se développer dans des niches d'investissements et sur le modèle institutionnel presque exclusif de la start-up (des milieux dans lesquels vont d'ailleurs s'intégrer des gens du MRC à partir de la fin des années 1970). Il fréquente des secteurs d'activité (industrie pétrolière, grands industriels de la chimie) dans lesquels opère un modèle plus ancien où « des entreprises bien établies $\{\ldots\}$ absorbent les découvertes de professeurs inventifs » (alors que rares sont les industries bien établies à vouloir risquer massivement des capitaux dans les biotechs et l'informatique/électronique - pas l'industrie pharmaceutique traditionnelle, par exemple, "souvent peu aventurière intellectuellement et financièrement conservatrice", comme l'explique Daniel Greenberg) (Greenberg, 2007, p. 83).

Plusieurs questions restent en suspens. Au sujet de l'expertise environnementale, d'abord. Depuis les années 1980, de larges communautés scientifiques et des grands groupes internationaux d'expertise et de production de connaissances sur l'environnement global GIEC (Groupe d'Experts Intergouvernemental sur l'Évolution du Climat), IGBP (International Geosphere-Biosphere Programme), etc. - se sont constitués. Ces reconfigurations institutionnelles ont-elles complètement annihilé la légitimité d'un individu seul à s'exprimer sur la nature de la Terre ? En outre, quelles stratégies les scientifiques de l'ancienne et de la nouvelle générations adoptent-ils pour accroître le poids de leur parole politique dans le débat public sur la crise environnementale ? Ensuite, de nombreuses études restent à mener au sujet de l'entrepreneuriat scientifique : comment l'accueil de personnalités hybrides comme Lovelock a-t-il évolué dans la recherche publique et la recherche privée au cours des dernières décennies, notamment lorsque leurs savoirs contribuaient à alimenter des controverses sociotechniques sur l'environnement ou la santé ?; que nous apprennent les autres facettes d'inventeurs-théoriciens sur Lovelock et sur les reconfigurations des sciences de la nature ?; quels champs scientifiques ont vu prospérer le modèle " d'expert-théoricien » ou de «consultant-universitaire » avec un pied dans la recherche publique et l'autre dans le privé ?

\section{Remerciements}

\footnotetext{
${ }^{36}$ Cette mutation est d'abord un saut quantitatif au sein de pratiques market-oriented existantes (voir Greenberg, 2007 \& Mercelis et al., 2017). Elle est également qualitative, avec des reconfigurations institutionnelles, juridiques et territoriales (les campus de type Silicon Valley - qui n'ont d'ailleurs jamais attiré un Lovelock qui leur préfère la petite structure familiale).
} 
Nous souhaitons remercier Amy Dahan, Sebastian Grevsmühl et Yannick Mahrane pour leur relecture d'une version préliminaire de ce texte. L'un d'entre nous (Sébastien Dutreuil) voudrait également remercier: les acteurs scientifiques ayant accepté des entretiens (notamment James Lovelock, Timothy Lenton, Andrew Watson); Katherine Ford, Katie Lissamore, Prabha Shah et Nick Wyatt travaillant au Science Museum de Londres pour leur aide précieuse à l'occasion d'un travail sur les archives de Lovelock; Jean Gayon et Philippe Huneman d'avoir encadré la thèse de doctorat dont est issue la contribution de l'auteur au présent texte. 


\section{REFERENCES BIBLIOGRAPHIQUES}

\section{Sources non publiées}

Archives de James Lovelock, 1935 - 2011, 78 boîtes, Science Museum Library and Archives, Science Museum at Wroughton, Hackpen Lane, Wroughton, Angleterre.

\section{Sources publiées}

BENNINGHOFF, M. (2011), «Publish or perish!" : la fabrique du chercheur-entrepreneur, Carnets de bord, $\mathrm{n}^{\circ} 17,47-58$

BIAGIOLI, M. (1993), Galileo courtier: The practice of science in the culture of absolutism, University of Chicago Press

BOUTILLIER, S. (2003), L'entrepreneur scientifique ou la dévalorisation du travail de chercheur, Innovations, 2003/1, n 17, 79-103

BRIDAY, R. (2014), Une histoire de la chimie atmosphérique globale. Enjeux disciplinaires et d'expertise de la couche d'ozone et du changement climatique, thèse de doctorat, École des Hautes Études en Sciences Sociales / Centre Alexandre Koyré, Paris

CHADAREVIAN, S. (de) (2011), The Making of an Entrepreneurial Science Biotechnology in Britain, 1975-1995, Isis, 102/4, 601-633

CHARLSON, R., LOVELOCK, J., ANDREAE, M., WARREN, S. (1987), Oceanic phytoplankton, atmospheric sulphur, cloud albedo and climate, Nature, 326(6114), 655-661 CONWAY, E. (2008), Atmospheric Science at NASA: a history, Johns Hopkins University Press.

DOEL, R.E. (2003), Constituting the Postwar Earth Sciences The Military's Influence on the Environmental Sciences in the USA after 1945, Social Studies of Science, 33(5), 635 - 666 DUTREUIL, S. (2017), James Lovelock, Gaïa et la pollution : un scientifique entrepreneur à l'origine d'une nouvelle science et d'une philosophie politique de la nature, Zilsel, 2, 19-61 DUTREUIL, S. (2016), Gaïa : hypothèse, programme de recherche pour le système Terre, ou philosophie de la nature? Thèse de doctorat, Université Paris 1 Panthéon-Sorbonne, Paris EDWARDS, P.N. (2010), A vast machine: Computer models, climate data, and the politics of global warming, The MIT Press.

FRIEDMAN, R. (1989), Appropriating the weather: Vilhelm Bjerknes and the construction of a modern meteorology, Ithaca: Cornell University Press

GALVEZ-BEHAR, G. (2016), Louis Pasteur, entrepreneur. Pour une histoire économique des mondes savants, <halshs-01267638v4>, Working paper, https://halshs.archivesouvertes.fr/halshs-01267638/

GREENBERG, D. (2007), Science for sale. The perils, reward, and delusions of campus capitalism, University of Chicago Press

GREVSMÜHL, S. (2012), À la recherche de l'environnement global : de l'Antarctique à l'espace et retour: instrumentations, images, discours et métaphores, thèse de doctorat, École des Hautes Études en Sciences Sociales, Paris.

HITCHCOCK, D.R., LOVELOCK, J. (1967), Life detection by atmospheric analysis, Icarus, 7(1-3), 149-159

KEITH, S.T. (1981), Inventions, Patents and Commercial Development from Governmentally Financed Research in Great Britain: The Origins of the National Research Development Corporation, Minerva, 19/1

LATOUR, B. (2015), Face à Gaïa : huit conférences sur le nouveau régime climatique, La Découverte. 
LOVELOCK, J. (2010) (2010-04-07), Oral history of British science (site web), "Lovelock, James (part 5 of 13). An Oral History of British Science", http://sounds.bl.uk/Oralhistory/Science/021M-C1379X0015XX-0005V0, minutes 20'05-31'20 (consulté le 03.04.2018)

LOVELOCK, J. (2009), The Vanishing Face of Gaia, Basic Books

LOVELOCK, J. (2006), The revenge of Gaia, Penguin Books

LOVELOCK, J. (2000), Homage to Gaia: the life of an independent scientist, Oxford University Press

LOVELOCK, J. (1995), The ages of Gaia: a biography of our living earth, Second Edition, Oxford University Press

LOVELOCK, J. (1979a), Gaia: a new look at life on Earth, Oxford University Press

LOVELOCK, J. (1979b), The independent practice of science, New Scientist, September 6, 714-717

LOVELOCK, J. (1972), Gaia as seen through the atmosphere, Atmospheric Environment, 6, 579-580

LOVELOCK, J. (1971), Air pollution and climatic change, Atmospheric Environment, 5(6), 403-411

LOVELOCK, J., MAGGS, R., RASMUSSEN, R. (1972), Atmospheric dimethyl sulphide and the natural sulphur cycle, Nature, 237, 452-453

LOVELOCK, J., MAGGS, R., WADE, R. (1973), Halogenated hydrocarbons in and over the Atlantic, Nature, 241, 194-195

LOVELOCK, J., PENKETT, S.A. (1974), PAN over the Atlantic and the smell of clean linen, Nature, 249, 434

MASSARD-GUILBAUD, G. (2003), Culture, technique, gestion de l'espace. Une histoire sociale de la pollution industrielle dans les villes françaises (1789-1914), Thèse HDR, Lyon II.

MERCELIS, J., GALVEZ-BEHAR, G., GUAGNINI, A. (2017), Commercializing science: nineteenth- and twentieth-century academic scientists as consultants, patentees, and entrepreneurs, History and Technology, 33:1, 4-22

MESSEGHEM, K., SAMMUT, S. (2011), L'entrepreneuriat, Editions EMS

NICHOLAS, T. (2014), Technology, Innovation and Economic Growth in Britain Since 1870, Working paper, http://www.people.hbs.edu/tnicholas/tech_cehb.pdf

ORESKES, N., CONWAY, E. (2010), Merchants of Doubt: How a Handful of Scientists Obscured the Truth on Issues from Tobacco Smoke to Global Warming, Bloomsbury Press.

PRINN, R.G., SIMMONDS, P.G., RASMUSSEN, R.A., ROSEN, R.D., ALYEA, F.N., et al. (1983), The atmospheric lifetime experiment: 1. Introduction, instrumentation, and overview, Journal of Geophysical Research: Oceans, 88(C 13), 8353-8367

SHAPIN, S. (2008), The Scientific Life: A Moral History of a Late Modern Vocation, Chicago University Press

STEWART, L. (1997), La ville de Londres. La rencontre de la science et du marché, La Recherche, $\mathrm{n}^{\circ}$ 300, juillet-août 1997, pp. 34-39

TURCHETTI, S., ROBERTS, P. (2014), The surveillance imperative: geosciences during the cold war and beyond, Palgrave Macmillan. 